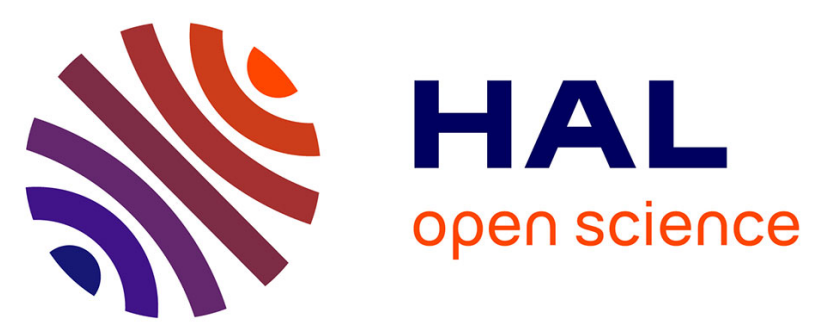

\title{
An advanced underplatform damper modelling approach based on a microslip contact model
}

Luca Pesaresi, Jason Armand, Christoph Schwingshackl, Loïc Salles, Chian W Wong

\section{- To cite this version:}

Luca Pesaresi, Jason Armand, Christoph Schwingshackl, Loïc Salles, Chian W Wong. An advanced underplatform damper modelling approach based on a microslip contact model. International Symposium on Transport Phenomena and Dynamics of Rotating Machinery (ISROMAC 2017), Dec 2017, Maui, United States. hal-02369256

\section{HAL Id: hal-02369256 \\ https://hal.science/hal-02369256}

Submitted on 18 Nov 2019

HAL is a multi-disciplinary open access archive for the deposit and dissemination of scientific research documents, whether they are published or not. The documents may come from teaching and research institutions in France or abroad, or from public or private research centers.
L'archive ouverte pluridisciplinaire HAL, est destinée au dépôt et à la diffusion de documents scientifiques de niveau recherche, publiés ou non, émanant des établissements d'enseignement et de recherche français ou étrangers, des laboratoires publics ou privés.

\section{(c)(1)}

Distributed under a Creative Commons Attribution| 4.0 International License 


\title{
An advanced underplatform damper modelling approach based on a microslip contact model
}

\author{
L.Pesaresi $^{1}{ }^{*}$, J.Armand ${ }^{1}$,C.W.Schwingshackl ${ }^{1}$, L.Salles ${ }^{1}$, C.Wong ${ }^{2}$
}

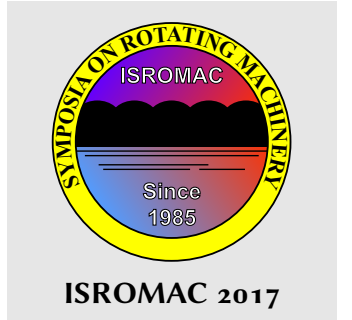

International

Symposium on

Transport Phenomena

and

Dynamics of Rotating

Machinery

Maui, Hawaii

December 16-21, 2017

\begin{abstract}
High-cycle fatigue caused by large resonance stresses remains one of the most common causes of turbine blades failures. Friction dampers are one of the most effective and practical solutions to limit the vibration amplitude, and shift the resonance frequencies of the turbine assemblies far from operating speeds. However, predicting with good accuracy the effects of underplatform dampers on the blades dynamics, still represents a major challenge today, due to the complex nature of the nonlinear forces at the interface, characterised by transitions between stick, slip, and separation conditions. The most common modelling approaches developed recently are based on the explicit FE model for the damper, and on a dense grid of $3 \mathrm{D}$ contact elements comprised of Jenkins elements, or on a single $2 \mathrm{D}$ microslip element on each surface. In this paper, a combination of the two approaches is proposed. A $3 \mathrm{D}$ microslip element, based on a modified Valanis model is proposed and a series of these elements are used to describe the contact interface. The proposed model and its predicting capabilities are then evaluated against a simplified blade-damper model, based on an underplatform damper test rig recently developed by the authors. A comparison with a more simplistic modelling approach based on macroslip contact elements, highlights the improved accuracy of the new model to predict the experimental nonlinear response.

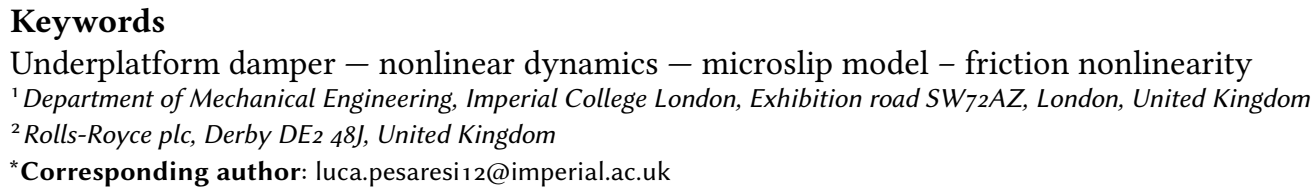

\section{INTRODUCTION}

\section{NOMENCLATURE}

$\tilde{\mathbf{F}}_{x}, \tilde{\mathbf{F}}_{y}, \tilde{\mathbf{F}}_{z}$ Contact force coefficients

$\tilde{\mathrm{X}}, \tilde{\mathrm{Y}}, \tilde{\mathrm{Z}}$ Contact displacement coefficients

$\mathbf{F}_{n l}, \mathbf{P} \quad$ Nonlinear forces, external forces.

Q Harmonic displacements

$\mathrm{Z}(\omega) \quad$ Dynamic stiffness matrix

$\mu \quad$ Friction coefficient

$v \quad$ Poisson ratio

E Young's modulus

$E_{0}, E_{t} \quad$ Initial loading slope, macroslip slope

$F, F_{z} \quad$ Tangential friction force, normal contact force

$k \quad$ Parameter controlling microslip level

$K_{z z} \quad$ Discrete influence coefficients

M.C.K Mass, damping and stiffness matrices

p Pressure distribution

$q \quad$ Tangential relative displacement
One of the most common causes of failure for gas turbines is high-cycle fatigue (HCF) driven by large resonant stresses [1]. Underplatform dampers are devices used by aeroengine manufacturers to reduce the risk of HCF failure, since they dissipate the vibrational energy while at the same time acting as a seal between blades to increase the thermodynamic efficiency $[2,3]$. Due to the nonlinear nature of the contact forces, characterised by stick-slip and separation, the dynamic behaviour of the blades becomes very nonlinear making its analysis much more complex. Continuous research has been conducted in this field for the development of new numerical damper models which attempted to improve the fidelity and/or computational cost $[4,5,6,7]$. Most of the modelling approaches proposed rely on frequency domain solvers based on the multi-harmonic balance method in conjunction with continuation techniques, as they represents an efficient approach when only the steady-state response is of interest $[8,9,10]$. Various contact models have been used to simulate the interaction between the platform and the blades, which can be classified as macroslip models $[11,12,13,14]$ or microslip models $[3,15,16]$. Initially, the macroslip contact models were only $1 \mathrm{D}[11]$, and subsequently they were extended to allow the relative normal motion between the damper and platform $[5,12,14]$. In [13], a fully coupled 3D macroslip contact model, capable of simulating a coupled in-plane $2 \mathrm{D}$ motion and the normal relative motion was developed. With regards to the microslip models, various 
concepts have been developed $[15,16,17]$ with the most common being a parallel elasto-plastic springs arrangement, initially proposed by Iwan in [18] for $1 \mathrm{D}$ tangential motion, and then recently extended to allow relative normal motion in $[19,20]$. When a microslip contact model is employed, normally only a single element describes the behaviour of the whole contact interface and specifically-designed experimental set-ups are used to tune the element $[3,21,22]$. This approach works well for non-conforming contact surfaces (cylindrical dampers), since the contact is normally very localised. However, it could lead to an over-simplification for conforming contacts (wedge dampers) since the kinematics of the contact, which is partially lost using one single microslip element, plays a significant role on the nonlinear dynamic behaviour [23]. A different approach to model microslip, which allows an improved description of the contact kinematics, is to discretise the contact area with a grid of macroslip contact elements $[6,7,24,25]$. When this approach is chosen, a significant microslip behaviour can be observed only when a pressure gradient is present at the contact interface as shown in [24]. However, in real contacts, frictional dissipation can also occur with a nominally uniform pressure distribution, since on the scale of the asperities no contact is truly conforming due to the surface roughness [26]. For this reason, the overall microslip behaviour observed could be seen as the combination of two microslip processes with different scales, a larger scale microslip due to non-uniform pressure distribution, and the microscale microslip at the asperity level. In this study a new modelling approach is presented, which is characterised by the discretisation of the contact area with a grid of contact elements, which are tuned to reproduce the microscale energy dissipation. For this scope, a new $3 \mathrm{D}$ microslip contact model based on a modified Valanis elasto-plastic model $[27,28]$ was used, and it was then tuned numerically using a semi-analytical contact solver [29]. The modelling approach was then evaluated against an underplatform damper test rig recently developed by the authors $[24,30]$, showing some promising capabilities for the prediction of the nonlinear dynamics of the blades.

\section{MODELLING APPROACH}

The modelling approach proposed in this paper is based on the previous work presented in $[4,24]$, but introduces a new contact element and attempts to increase the fidelity of the contact description at the platform-damper interface. This proposed approach is well suited to model wedge dampers, as well as other structures which have friction nonlinearities caused by conforming contacts. The damper is included in the model explicitly, using an FE description which allows to account for its flexibility and inertia properties. This aspect is important for dampers which are relatively flexible and that operate close to a "stuck" condition, since neglecting the local flexibility might lead to inaccuracies in the calculation of the contact forces.

The damper and the blades FE models are then coupled using a series of contact elements newly developed and de- scribed in details in the next section. To allow an accurate description of the contact properties, the damper-platform interface is discretised with a grid of contact elements, each of those representing the contact behaviour of a portion of the contact as shown in Fig.1. Due to the manufacturing tolerances, even nominally conforming surfaces can be characterised by a lack of conformity which, together with non-uniform normal loading might lead to a non-uniform pressure distribution as shown in the example of Fig.1. This variation of pressure is taken into account by explicitly defining a different initial pre-load on each element depending on its position on the contact interface, and on the area of the associated patch. The microslip behaviour on the microscale level, caused by the surface roughness, as well as the contact stiffness associated with each portion of the contact, is taken into account by tuning the parameters of each contact element, as described in section 4 .

The numerical analysis is based on an exisiting multiharmonic balance (MHBM) solver with continuation coupled with a model reduction technique, included in the code FORSE (FOrced Response SuitE) and discussed in detail in $[4,14,31]$. Due to the friction forces arising at the contact interface, the equations of motion for the blades and damper are nonlinear, and can be written in the following form:

$$
\mathbf{M x}(t)+\mathbf{C x}(t)+\mathbf{K x}(t)+\mathbf{F}_{n l}(\mathbf{x}(t), \mathbf{x}(t))=\mathbf{P}(t) .
$$

where $\mathbf{M}, \mathrm{C}, \mathrm{K}$ are the mass, damping, and stiffness matrices. $\mathbf{P}$ are the external excitation forces and $\mathbf{F}_{n l}$ are the nonlinear contact forces dependent on the relative motion of the interacting nodes at the interface. According to the MHBM, the response for each DOF of the system can be expressed as a Fourier series truncated at the $n^{\text {th }}$ harmonic:

$$
\mathbf{q}(\mathbf{t})=Q_{0}+\sum_{j=1}^{n} \mathrm{Q}_{j}^{c} \cos m_{j} \omega t+\mathrm{Q}_{j}^{s} \sin m_{j} \omega t
$$

where $\mathrm{Q}_{0}, \mathrm{Q}_{j}^{c}$ and $\mathrm{Q}_{j}^{s}$ are the harmonic coefficients for each DOFs. When equation 2 is inserted in equation 1 , and the harmonic terms balanced with a Galerkin projection, a system of equations to determine all harmonic components can be obtained in the frequency domain as follows:

$$
Z(\omega) Q+F(Q)-P=0
$$

where $\mathrm{Q}=\left\{\mathrm{Q}_{0}, \mathrm{Q}_{1}^{c}, \mathrm{Q}_{1}^{s}, \ldots, \mathrm{Q}_{n}^{c}, \mathrm{Q}_{n}^{s}\right\}^{T}$ is a vector of harmonic coefficients, $F(Q)$ is the vector of nonlinear forces and $\mathbf{P}$ is the external excitation. $\mathbf{Z}(\omega)$ is the dynamic stiffness matrix of the system. To reduce the size of the problem, a model reduction based on a FRF matrix representation is applied [31], and the harmonic coefficients are then calculated with a Newton-Raphson iterative solver.

\section{3D MICROSLIP CONTACT ELEMENT}

A new contact element, which enables to simulate $3 \mathrm{D}$ contact motion while taking into account the microslip effects due 


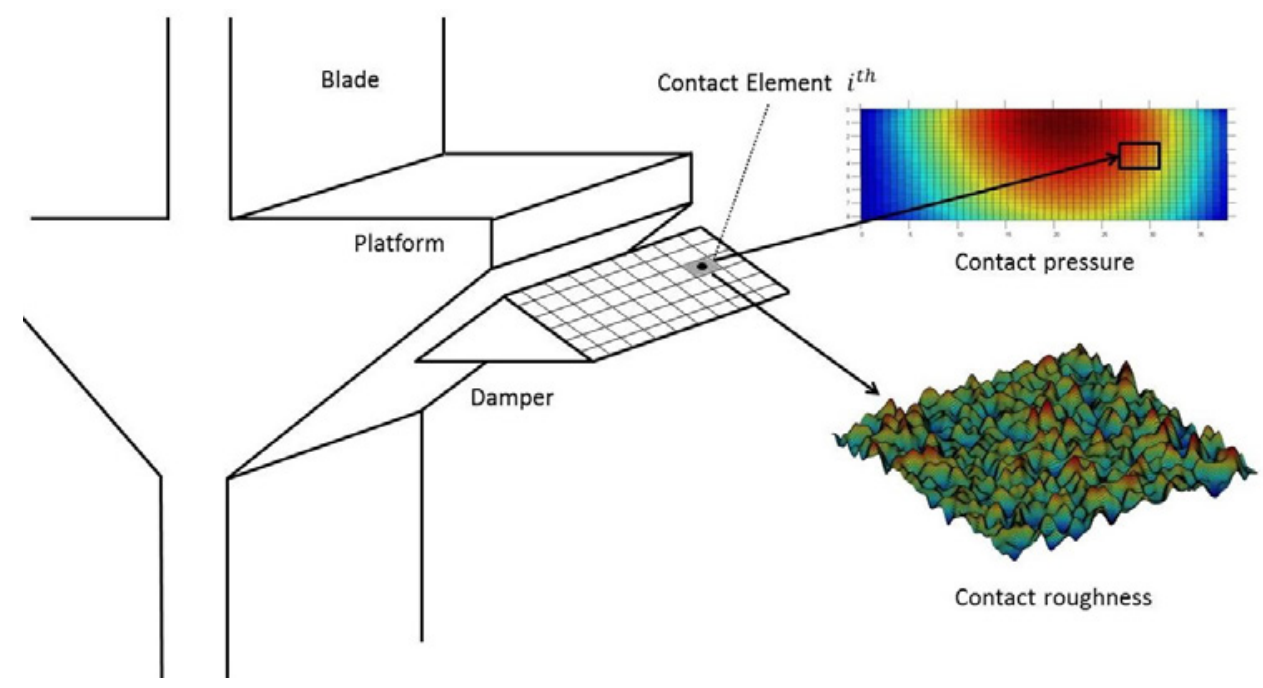

Figure 1. Discretisation of the damper-platform contact interface.

to surface roughness was used for the present analysis and implemented in the nonlinear dynamic code FORSE. This element is based on the Valanis model, originally developed to describe the elasto-plastic behaviour of materials under cylic loading conditions [27]. The Valanis model was then succesfully employed in [28] to model the frictional hysteretic behaviour of a lap joint subject to harmonic excitation. In the present study, the model was modified to allow the inclusion of a variable time-dependent normal load $F_{z}(t)$ as originally proposed in [32]. With this approach, the friction limit is time-dependent allowing a full coupling between the tangential and normal relative displacements at the contact. Therefore, the tangential friction force is defined as follows:

$$
\dot{F}=E_{0} \dot{q} \frac{\left[1+\frac{\lambda}{E_{0}} \frac{\dot{q}}{|q|}\left(E_{t} q-F\right)\right]}{\left[1+k \frac{\lambda}{E_{0}} \frac{\dot{q}}{|q|}\left(E_{t} q-F\right)\right]}=f(q, \dot{q}, F, \lambda)
$$

where $E_{0}$ corresponds to the tangential contact stiffness of the initial loading, $E_{t}$ is the slope of the macroslip region, $k$ is a parameter which characterises the microslip transition, $q$ is the contact tangential displacement, and $\lambda$ is a parameter defined as:

$$
\lambda=\frac{E_{0}}{\mu F_{z}(t)\left(1-k \frac{E_{t}}{E_{0}}\right)}
$$

One of the advantage of the Valanis formulation is that, by defining a positive value for $E_{t}$, it permits to reproduce contact hysteresis loops where the macroslip region has a slope. This contact behaviour has been observed experimentally in friction joints that are subject to fretting wear, as reported in $[28,33]$.

To simulate a full $3 \mathrm{D}$ motion, two Valanis elements were used along the $x$ and $y$ direction of the local coordinate system defined for the element " $i$ " as shown in Fig.2. This allows

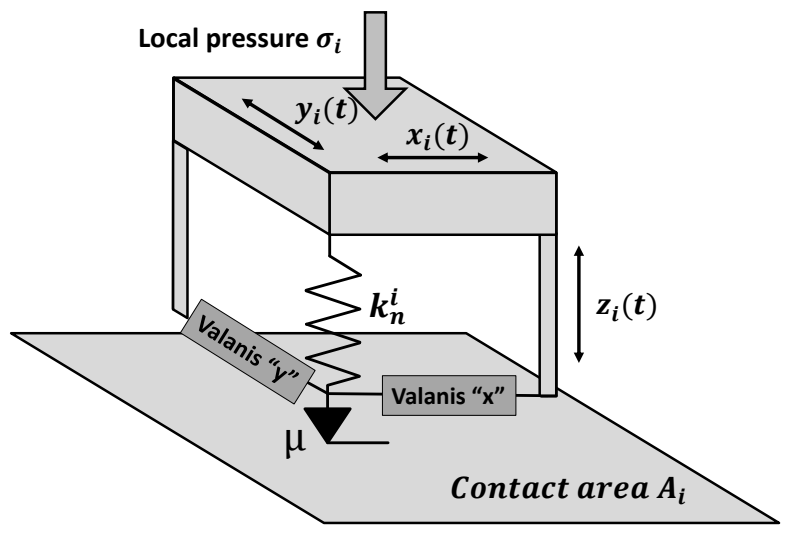

Figure 2. New 3D microslip element schematic.

do describe a $2 \mathrm{D}$ in-plane motion even if there is no coupling between the two directions. For each contact element " $i$ ", a set of parameters needs to be defined: $E_{0}, E_{t}, k$, the friction coefficient $\mu$, the normal contact stiffness $k_{n}$, and the initial element pre-load $N_{0}=\sigma_{i} A_{i}$, with $\sigma_{i}$ and $A_{i}$ being the local pressure and contact area associated with the element. In this study, a simplification was made assuming the parameters $E_{0}$ and $k_{n}$ constant with time, but the model is flexible to allow the inclusion of different load-dependent stiffness laws in the future.

An alternate frequency-time method (AFT) [8] is used to allow an easier calculation of the contact forces which are nonlinear with displacements, using a separate routine in the time-domain. A simplified flow-chart for the contact forces calculation is shown in Fig.3.

The contact relative displacements $\tilde{X}, \tilde{Y}$ and $\tilde{Z}$ of the mating nodes between the platform and the damper are expressed as harmonic coefficients in the local coordinate system. An inverse Fourier transformation is then applied, and the non- 


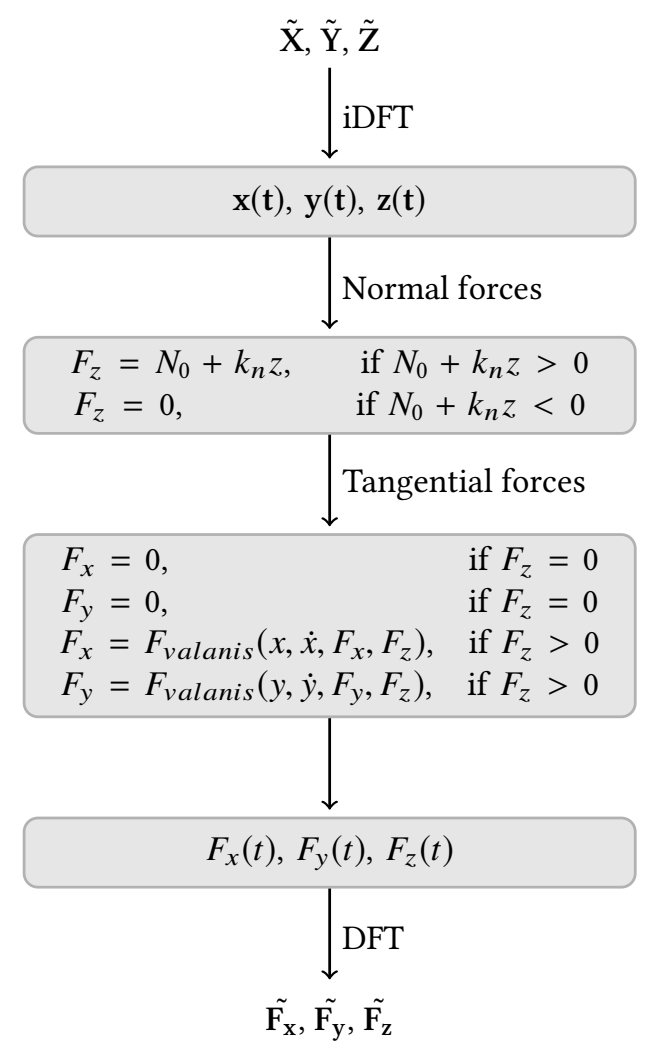

Figure 3. Flow-chart of the contact element routine.

linear contact forces are then evaluated, using an implicit Euler's method for the tangential forces. The contact forces for the three directions in the local coordinate system are then transformed back to the frequency-domain, $\tilde{\mathbf{F}_{x}}, \tilde{\mathbf{F}_{\mathbf{y}}}, \tilde{\mathbf{F}_{z}}$ and this force calculation is repeated until convergence is reached for the Newton-Raphson scheme. The parameter " $k$ ", which controls the level of "microslip" in the contact, can vary from "0" (infinite microslip) to "1" (no microslip), and the effect of this variation under a constant normal load is shown in Fig.4.

One of the drawbacks of using the Valanis model for friction contacts, is that unlike in other joint models, the initial loading slope, which corresponds to $E_{0}$ (point $\mathrm{A}$ in Fig.4), is different from the "re-loading slope" (point B in Fig.4). In fact, from equation 4 , for $E_{t}=0$, the re-loading slope is equal to $2 E_{0} /(1+k)$, and therefore it is always higher than $E_{0}$ apart from the limit case $k=1$, which is not defined. Since only the steady-state solution is of interest here, the initial load is always neglected, and for a correct analysis, the dependence of the contact stiffness on $k$ must be taken into account when specifying the input parameters.

To evaluate the capability of the new contact model under coupling conditions, a tangential harmonic motion coupled to an in-phase normal motion with a separation event is shown in Fig.5. As a result of this coupling, the tangential friction force is not constant anymore at the sliding limit, but follows the curve $\mu N(t)$. When the amplitude of the

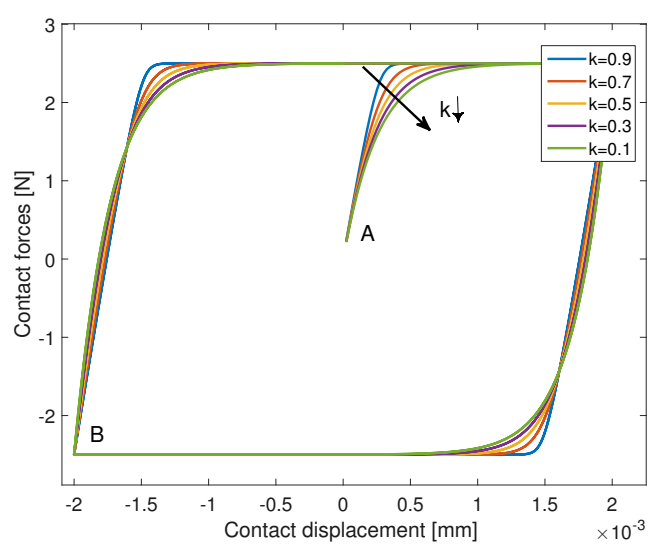

Figure 4. Contact hysteresis loops with varying microslip parameter " $k$ ".

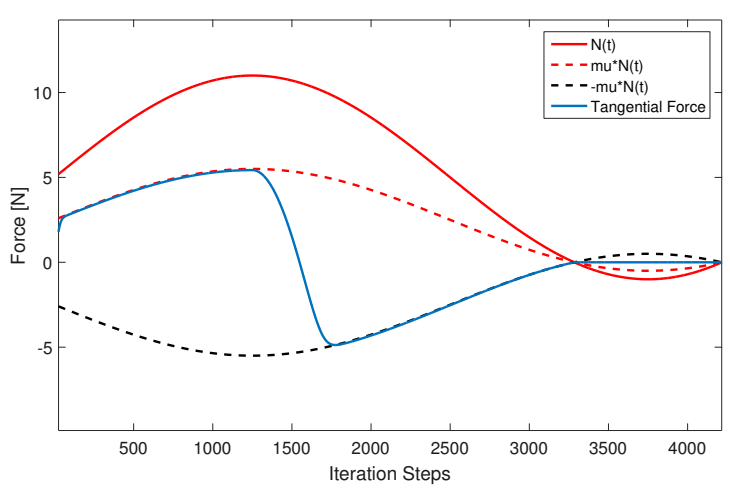

Figure 5. Tangential contact force calculated under varying normal load.

normal motion is increased, the contact hysteresis loops are progressively deformed, showing a flat part on the left side which corresponds to the separation event as shown in Fig.6.

\section{TEST CASE - UPD RIG}

To validate the proposed modelling approach, an underplatform damper (UPD) rig recently developed at Imperial College London (see $[24,30]$ ) was chosen as the main test case. The UPD rig is an experimental set-up that allows the investigation of the effect of UPDs on blade-like structures under a controlled lab environment. The assembly of the rig can be seen in Fig. 7 .

Two pseudo beam-like blades are fixed to a common base, simulating a rigid disk, and are clamped via a hydraulic cylinder to a large inertia block. The damper is a wedge type, [3], which has a triangular cross section with a characteristic angle. Unlike a real high pressure turbine blade, the aerofoil is replaced by a straight rectangular cross-section beam, but still maintaining vibration modes similar to a real blade. The centrifugal load is simulated via a pulley system with calibrated masses, and the excitation is provided by an electrodynamic shaker (Data Physics $V_{4}$ ) attached near 


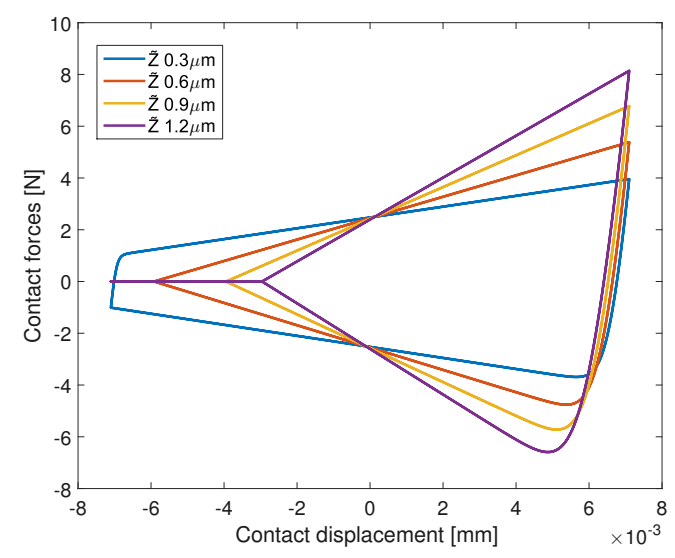

Figure 6. Contact hysteresis loops with increasing normal displacement.

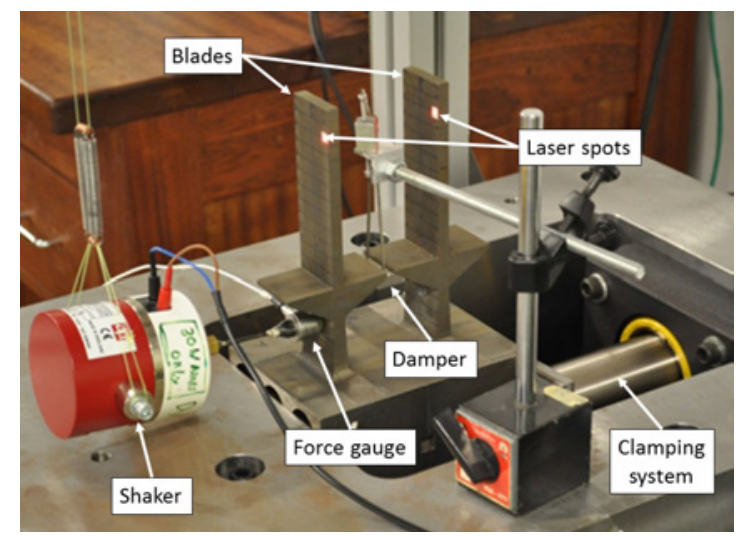

Figure 7. Underplatform damper test rig lab set-up.

the root of the blade to minimise its impact. A non-contact measurement system, which employs two single point laser Doppler vibrometers (Polytec OFV-503) was used to capture the dynamic response of both blades near the tip (see Fig.7). A stepped sine test was performed in a narrow frequency band around the mode of interest, always keeping the excitation force constant within a tolerance range to control the nonlinearity of the system. Different tests were performed with increasing excitation forces in order to progressively activate the friction nonlinearity at the damper-platform interface and characterise the dynamics of the system.

\section{CONTACT ELEMENT TUNING}

One of the fundamental steps of the proposed modelling approach is the tuning of the contact parameters for the newly-developed friction contact element. In fact, including more accurate information about the contact nonlinearity, which is the driver for the nonlinear behaviour of the system, is very important to improve the prediction capabilities of the proposed modelling approach. The approach proposed here for the contact element tuning is mainly based on a semi-analytical contact solver described in section 4.1 , but fully experimental approaches based on specifically-designed test set-ups could be used as well.

The advantage of using a semi-analytical contact solver relies on the possibility to simulate any rough surface, without any assumption on the statistical distribution of the asperities. In addition, the relatively high speed of computation allows to overcome the problems of FE rough contact simulations, which would require prohibitively large models to account for a good representation of the surface features.

\subsection{Semi-analytical contact solver based on the Boundary Element Method (BEM)}

A BEM-based contact solver, previously developed and validated by the authors $[29,34]$, is used here to perform a refined analysis of the damper-platform friction interface and tune the new contact element. The contact solver uses the projected conjugate gradient method [35] and a discreteconvolution fast Fourier transform to accelerate the computation. It assumes the elastic half-space body description, which makes it possible to use the Boussinesq and Cerruti potentials $[36,37]$ to compute the surface elastic deflections in the normal and tangential directions from the pressures and shear tractions in the contact area. Equation 6 gives the component of normal displacement $u_{z}$ due to a pressure distribution $p$. Equation 7 is the discretised form of Eq. 6 on a regular grid of $N_{x} \times N_{y}$ points. Similar equations are used to compute the the tangential displacements. The first step of the algorithm is to solve the normal contact problem using the conjugate gradient method, after which the tangential problem can be solved using the Coulomb friction law to bound the shear distribution in the slipping region.

$$
u_{z}(x, y)=\frac{1-v^{2}}{\pi E} \int_{-\infty}^{+\infty} \int_{-\infty}^{+\infty} \frac{p(\xi, \eta)}{\sqrt{(\xi-x)^{2}+(\eta-y)^{2}}} d \xi d \eta
$$

where $E$ and $v$ are the Young's modulus and Poisson ratio of the material, respectively.

$$
u_{z}(i, j)=K_{z z} \otimes p=\sum_{k=1}^{N_{x}} \sum_{l=1}^{N_{y}} p(k, l) K_{z z}(i-k, j-l)
$$

where $\otimes$ denotes the discrete convolution product and $K_{z z}(i, j)$ are the discrete influence coefficients [34] that give the normal displacement resulting from unit pressure on the element centred on the grid point $(i, j)$.

\subsection{Pressure distribution tuning}

Initially, a $3 \mathrm{D}$ non-contact topography-measuring interferometer Polytec TMS-100 was used to scan both contact surfaces of the damper used, revealing slightly curved profiles (see [24]). However, due to the limited dimensions of the scanning area of the interferometer, it was not possible to measure the surfaces of the platforms. Therefore, this limited the possibility to use the semi-analytical contact solver to obtain the contact pressure distribution, and an experimental approach was preferred instead. Fujifilm prescale pressure 
films were used at the damper-platform interface, and the prints obtained were then scanned and digitised in MATLAB. A $4^{\text {th }}$ order polynomial fit was used to approximate the pressure film prints (see Fig.8), which was then interpolated to obtain the input pre-loads for each contact element.
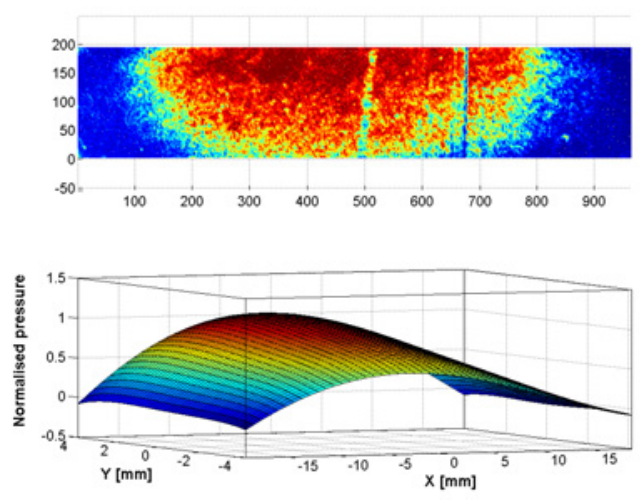

Figure 8. Pressure film digitised and approximated with a polynomial fit.

\subsection{Microscale tuning}

The semi-analytical contact solver described in section 4.1 was used to numerically tune the newly-developed contact element, allowing the model to reproduce the microslip effects due to the surface roughness, as well as the characteristic stiffness of the contact. Initially, a stylus profilometer was used for both the damper and the platforms to evaluate the roughness characteristics of the contact surfaces. A patch of $250 \mu m$ by $250 \mu m$ was generated in MATLAB, as shown in Fig.9, using a Gaussian distribution for the asperities which was able to reproduce the experimental roughness (with $R_{q}=0.177 \mu m$ and a correlation length $C l x=6.6 \mu m$ ). A contact mesh of 1024 by 1024 elements was used for the contact simulations, as it allows a good discretisation of the surface asperities, while keeping an acceptable computational time.

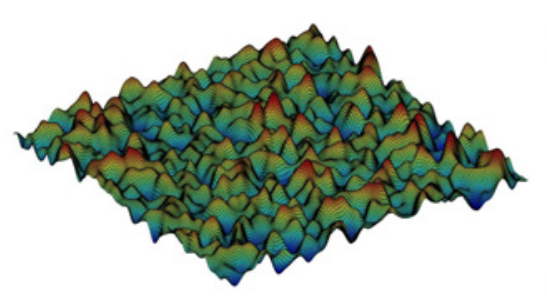

Figure 9. Simulated rough contact patch.

An initial simulation was performed under load-control and assuming a rough elastic contact, in order to obtain the values of the normal contact stiffness $k_{n}$ for different pressure levels, as shown in Fig.10. The data were obtained in a range of pressures between o to $10 \mathrm{MPa}$, since these are the values at the damper-platform interface under the $960 \mathrm{~N}$ damper load level used for the experiments and subsequent simulations.

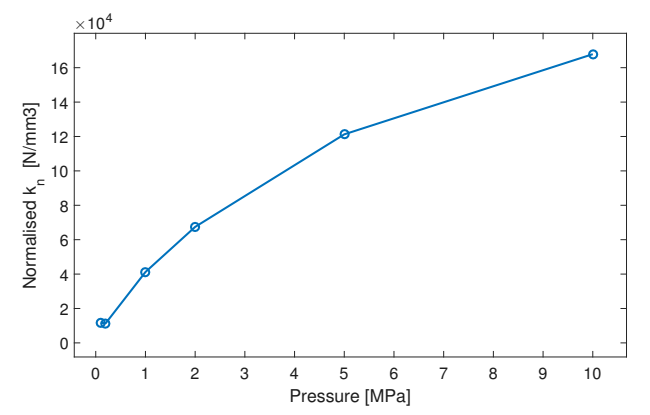

Figure 10. Variation of the parameter " $k_{n}$ " with contact pressure.

For the tangential problem, contact simulations were run imposing a sinusoidal displacement, and the corresponding contact hysteresis loops were obtained for different pressure levels (o.1 $\mathrm{MPa}$, $0.2 \mathrm{MPa}, 1 \mathrm{MPa}, 2 \mathrm{MPa}, 5 \mathrm{MPa}, 10 \mathrm{MPa}$ ) in the range of interest, as shown in Fig.11.

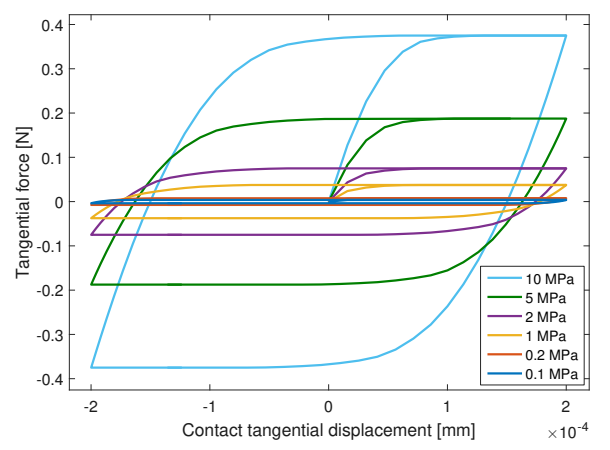

Figure 11. Simulated contact hysteresis loops at different pressure levels.

The friction Coulomb limit was set to be $\mu=0.6$, since this was the average value measured for the same material in previous experimental tests with a $1 \mathrm{D}$ friction rig developed at Imperial College London [38]. The hysteresis loops of Fig. 11 were then used to tune the contact element input parameters $E_{0}$ and $k$. Since both $E_{0}$ and $k$ influence the stiffness of the contact and its microslip transition, their selection is not trivial, and an optimisation was run in MATLAB based on the interior-point algorithm described in [39]. A set of parameters $E_{0}$ and $k$ could be found for each pressure level, which allowed the model to perfectly capture the numerical hysteresis loops. By interpolating the plots of $E_{0}$ and $k$ versus pressure (see Fig.12 and Fig.13), it was possible to specify a set of optimised parameters for each contact element at the damper-platform depending on the element pressure. A monotonic increasing trend was obtained for $E_{0}$, which indicates the tendency of the initial loading stiffness to increase at higher pressure, whereas a more complex behaviour characterised by a minimum close to $1 \mathrm{MPa}$ was obtained for the microslip parameter $k$. 


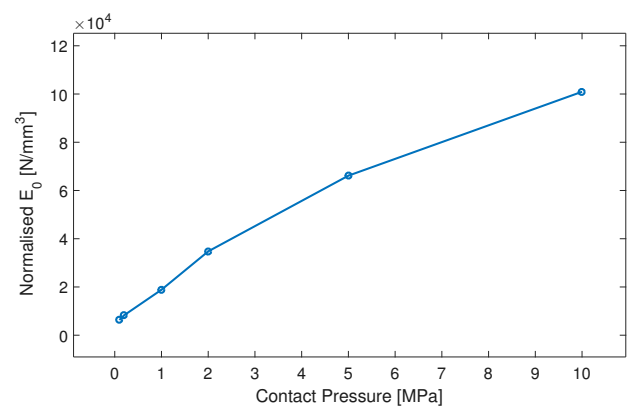

Figure 12. Variation of the parameter " $E_{0}$ " with contact pressure.

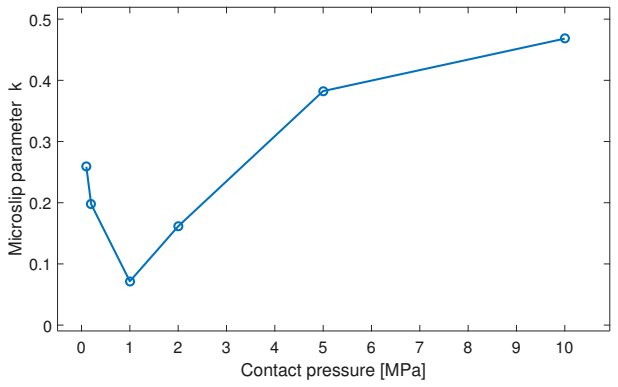

Figure 13. Variation of the microslip parameter " $k$ " with contact pressure.

\section{RESULTS AND DISCUSSION}

In this section, the prediction capabilities of the newly-proposed modelling approach are evaluated against the experimental data of the UPD rig, and are comapared to the previous model used in [24]. To allow a comparison with the previous results, the finite element model of the blades and damper were kept the same (see Fig.14) as in [24], as well as the subset of contact nodes used to discretise the damper-platform interface (see Fig.15). In addition, similarly to the previous model, it was decided to use the pressure distribution interpolated from the pressure films, as described in section 4.2. Therefore, the difference obtained with the previous model will allow to evaluate the contribution of the newly-developed contact element, as well as the tuning procedure described in section 4.3 for a higher fidelity description of the contact properties. In fact, in the previous model [24], the contact stiffness was assumed the same $\left(60000 \mathrm{~N} / \mathrm{mm}^{3}\right)$ for all the contact elements, based on previous measurements performed on the $1 \mathrm{D}$ friction rig described in [38].

The mode investigated for the present study is the first flexural out-of-phase (OOP) mode shown in Fig.14, as the damper operates close to a microslip condition, whereas the in-phase mode is dominated by a large separation at the contact interface (see [24]), and it is therefore much less interesting for the evaluation of the new approach. Some initial experiments were performed for the first flexural mode without the damper, in order to evaluate the background damping caused by the material and set-up configuration including the

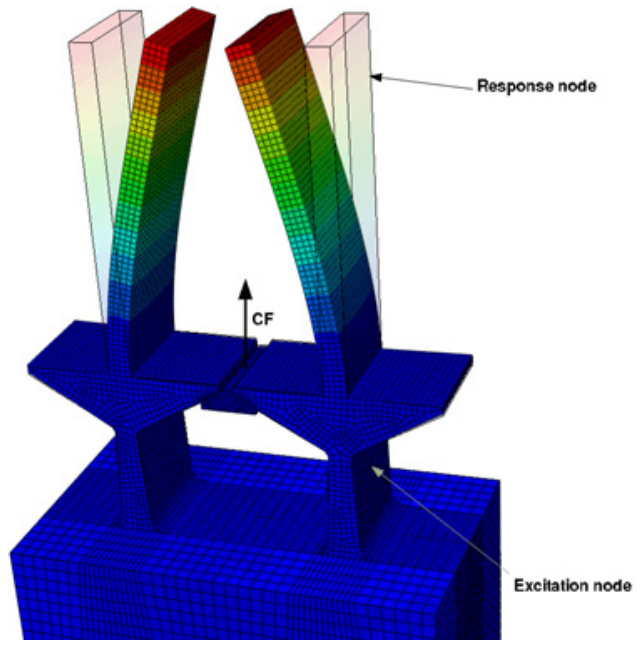

Figure 14. First flexural OOP mode of the blades and damper.

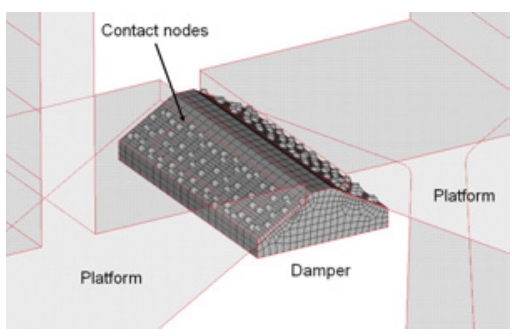

Figure 15. Contact friction elements at the damper-platform interface.

shaker. A loss factor of $\eta=0.04 \%$ was measured and it was included as modal damping in the linear model. The nonlinear measurements with the damper in place were performed using a constant damper load of $960 \mathrm{~N}$, which allowed a good conformity at the contact interface, and an increasing shaker excitation force from $0.096 \mathrm{~N}$ up to $17 \mathrm{~N}$ (shaker limit). The nonlinear FRFs of the experiments, new modelling approach, and old modelling approach are all compared in Fig.16, and the excitation and response location can be seen in Fig.14.

Thanks to an improved description of the stiffness distribution at the contact interface, the resonance frequency of the nearly-linear excitation case for the new model is slightly closer to the experiments, being $0.92 \%$ higher compared to the $1.01 \%$ of the previous model. Both models tend to overestimate the resonance frequency of the experimental OOP mode, which lies at $435.8 \mathrm{~Hz}$. A possible explanation for this behaviour could be an overestimation of the contact area in the model caused by the uncertainty in the polynomial approximation of the experimental pressure films. With regards to the amplitude estimation, the new model significantly improves the prediction accuracy compared to the previous model. In Fig.17, the nonlinear peak FRFs of the experiments and the two models are compared for the various excitation levels. Both models can capture the decreasing trend, but the new approach is much closer to the experimental curves 


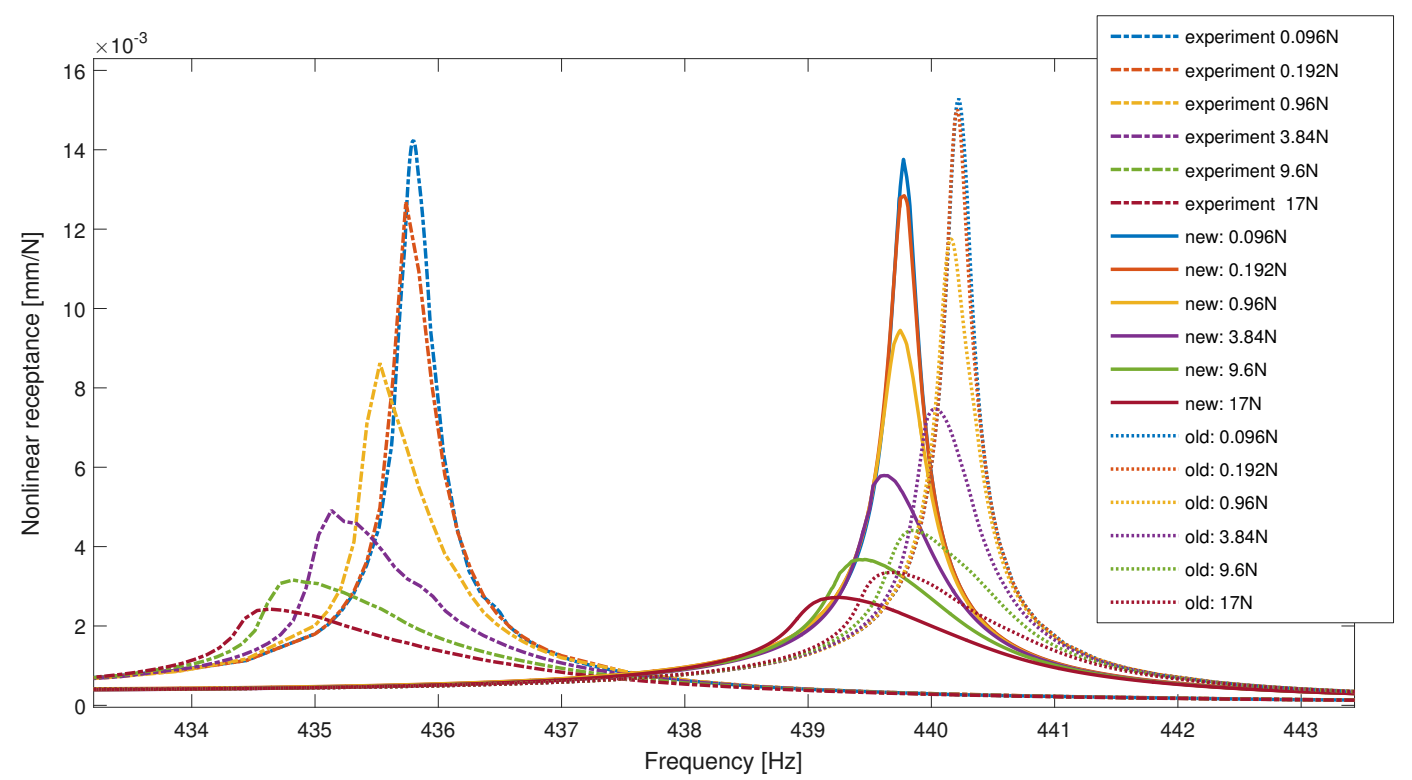

Figure 16. Nonlinear FRFs at increasing excitation levels.

having an amplitude percentage difference of $-3.3 \%,+1.2 \%$, $+8.89 \%,+17.91 \%,+16.28 \%,+11.9 \%$ for the increasing excitation levels compared to the $+7.3 \%,+18.3 \%,+36.2 \%,+52 \%,+39.5 \%$ and $+38.1 \%$ of the previous model.

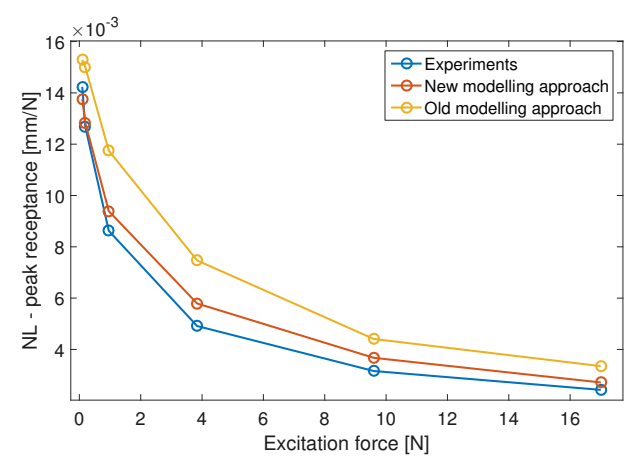

Figure 17. Comparison of peak nonlinear amplitudes at increasing excitation levels.

Therefore, taking into account the microscale miscroslip dissipation due to the surface roughness with a new microslip element lead to a significantly improved amplitude estimation, highlighting the promising capabilities of the new model for accurate dynamic predictions. The slight overestimation of the experimental nonlinear amplitudes observed is still acceptable, since factors such as the additional damping which might have been introduced by the damper pulling wire were not included in model. More investigations need to be performed for an improved evaluation of the damper-platform contact area, since this is one of the main factors for an accurate prediction of the modes resonance frequencies when the damper is in place.

\section{CONCLUSIONS}

In this study, a new modelling approach for underplatform dampers was presented and evaluated against the experimental data of a recently-developed test rig. The new model employs a dense discretisation of the damper-platform interface with a grid of $3 \mathrm{D}$ microslip contact elements. For this scope, a new 3D contact element based on a modified Valanis model, was developed and preliminary tests highlighted its capabilities for representing strong contact nonlinearities. The key idea of the model proposed, is to capture both the contribution of a macroscale microslip, due to non-uniform pressure distribution, as well as the microscale microslip due to the surface roughness. A tuning procedure for the microslip elements is also proposed, based on the numerical simulations of the normal and tangential behaviour of a representative contact patch having similar roughness characteristics to the real contact. The new modelling approach proved to be very effective in capturing the amplitude levels of the nonlinear FRFs, leading to a significant improvement compared to the previous models based on the use of macroslip contact elements. These findings suggest the relevance of the microscale dissipation, as well as of the improved description of the contact stiffness, in the overall frictional dissipation of the damper. In addition, the modelling approach proposed, only relies on the measurement of some contact properties, such as the friction coefficient and roughness, and therefore it represents a promising tool for the prediction of the nonlinear dynamic behaviour of blades constrained by friction dampers.

\section{ACKNOWLEDGMENTS}

The authors are grateful to Innovate UK and Rolls-Royce plc for providing the financial support for this work and 
for giving permission to publish it. This work is part of a collaborative R\&T project SILOET II P 19.6 which is co-funded by Innovate UK and Rolls-Royce plc and carried out by RollsRoyce plc and the Vibration UTC at Imperial College London.

\section{REFERENCES}

[1] B.A.Cowles. High cycle fatigue in aircraft gas turbine an industry prospective. International fournal of Fracture, 80:147-163, 1996.

[2] J. H. Griffin. A review of friction damping of turbine blade vibration. International fournal of Turbo and Jet Engines, 7:297-307, 1990.

[3] K. Y. Sanliturk, D. J. Ewins, and A. B. Stanbridge. Underplatform Dampers for Turbine Blades: Theoretical Modeling, Analysis, and Comparison With Experimental Data. Journal of Engineering for Gas Turbines and Power, 123(4):919, 2001.

[4] E. P. Petrov. Explicit Finite Element Models of Friction Dampers in Forced Response Analysis of Bladed Disks. Journal of Engineering for Gas Turbines and Power, 130(2):022502, 2008.

[5] C. M. Firrone, S. Zucca, and M. M. Gola. The effect of underplatform dampers on the forced response of bladed disks by a coupled static/dynamic harmonic balance method. International fournal of Non-Linear Mechanics, 46(2):363-375, 2011.

[6] E. Cigeroglu, N. An, and C. H. Menq. Forced Response Prediction of Constrained and Unconstrained Structures Coupled Through Frictional Contacts. Journal of Engineering for Gas Turbines and Power, 131(2):919, 2008.

[7] L. Panning, W. Sextro, and K. Popp. Spatial Dynamics of Tuned and Mistuned Bladed Disks with Cylindrical and Wedge-Shaped Friction Dampers. International Journal of Rotating Machinery, 9:219-228, 2003.

[8] M. Krack, L. Salles, and F. Thouverez. Vibration prediction of bladed disks coupled by friction joints. Archives of Computational Methods in Engineering, pages 1-48, 2016.

[9] M. Krack, L. Panning, and J. Wallaschek. A high-order harmonic balance method for systems with distinct states. Journal of Sound and Vibration, 332(21):5476 5488, 2013.

[10] L. Salles, B. Staples, N. Hoffmann, and C. W. Schwingshackl. Continuation techniques for analysis of whole aeroengine dynamics with imperfect bifurcations and isolated solutions. Nonlinear Dynamics, 86(3):1897-1911, 2016.

[11] J. H. Griffin. Friction Damping of Resonant Stresses in Gas Turbine Engine Airfoils. fournal of Engineering for Power, 102:329-333, 1980.

[12] B. D. Yang and C. H. Menq. Characterization of Contact Kinematics and Application to the Design of Wedge
Dampers in Turbomachinery Blading : Part 1 - StickSlip Contact Kinematics. Journal of Engineering for Gas Turbines and Power, 120(April 1998):410-417, 1998.

[13] B.D. Yang and C.H. Menq. Characterization of $3 \mathrm{~d}$ contact kinematics and prediction of resonant response of structures having $3 \mathrm{~d}$ frictional constraint. Fournal of Sound and Vibration, 217(5):909 - 925, 1998.

[14] E. P. Petrov and D. J. Ewins. Analytical Formulation of Friction Interface Elements for Analysis of Nonlinear Multi-Harmonic Vibrations of Bladed Disks. Journal of Turbomachinery, 125(2):364, 2003.

[15] C. H. Menq, J. Bielak, and J.H. Griffin. The influence of microslip on vibratory response, part I: A new microslip model. Fournal of Sound and Vibration, 107(2):279-293, June 1986.

[16] G. Csaba. Forced Response Analysis in Time and Frequency Domains of a Tuned Bladed Disk With Friction Dampers. Journal of Sound and Vibration, 214(3):395412, July 1998.

[17] K.Y. Sanliturk and D. J. Ewins. Modelling TwoDimensional Friction Contact And Its Application Using Harmonic Balance Method. fournal of Sound and Vibration, 193:511-523, 1996.

[18] W. D. Iwan. A distributed-element model for hysteresis and its steady-state dynamic response. Journal of Applied Mechanics, 33(4):893-900, 1966.

[19] M. Rajaei and H. Ahmadian. Development of generalized Iwan model to simulate frictional contacts with variable normal loads. Applied Mathematical Modelling, 38(15):4006 - 4018, 2014

[20] C. Gastaldi and M. M. Gola. An Improved Microslip Model for Variable Normal Loads, pages 169-179. Springer International Publishing, Cham, 2015.

[21] G. Csaba. Modelling of a microslip friction damper subjected to translation and rotation. In Proceedings of ASME Gas Turbine and Aeroengine Congress and Exhibition, volume 4, page Voo4To3Ao12, 1999.

[22] C. Gastaldi and M. M. Gola. On the relevance of a microslip contact model for under-platform dampers. International fournal of Mechanical Sciences, 115-116:145 - 156, 2016.

[23] L. Pesaresi, M. Stender, V. Ruffini, and C. W. Schwingshackl. DIC Measurement of the Kinematics of a Friction Damper for Turbine Applications, pages 93-101. Springer International Publishing, Cham, 2017.

[24] L. Pesaresi, L. Salles, A. Jones, J.S. Green, and C.W. Schwingshackl. Modelling the nonlinear behaviour of an underplatform damper test rig for turbine applications. Mechanical Systems and Signal Processing, 85:662 - 679, 2017.

[25] C. M. Firrone and S. Zucca. Modelling friction contacts in structural dynamics and its application to turbine bladed 
disks. In f.Awrejcewicz, Numerical Analysis - Theory and Application, INTECH 2011, pages 301-334, 2011.

[26] C. Putignano, M. Ciavarella, and J.R. Barber. Frictional energy dissipation in contact of nominally flat rough surfaces under harmonically varying loads. Fournal of the Mechanics and Physics of Solids, 59(12):2442 - 2454, 2011.

${ }^{[27]}$ K. C. Valanis. Fundamental consequences of a new intrinsic time measure. plasticity as a limit of the endochronic theory. Arch. Mech., 32:171 - 191, 1980.

[28] L. Gaul and J. Lenz. Nonlinear dynamics of structures assembled by bolted joints. Acta Mechanica, 125(1):169$181,1997$.

[29] J. Armand, L. Salles, and C. W. Schwingshackl. Numerical simulation of partial slip contact using a semianalytical method. In ASME 2015 International Design Engineering Technical Conferences and Computers and Information in Engineering Conference, pages Voo8T13Ao22Voo8T13A022. American Society of Mechanical Engineers, 2015 .

[30] L. Pesaresi, L. Salles, R. Elliot, A. Jones, J. S. Green, and C. W. Schwingshackl. Numerical and experimental investigation of an underplatform damper test rig. Applied Mechanics and Materials, 849:1-12, 102016.

[31] E. P. Petrov. A High-Accuracy Model Reduction for Analysis of Nonlinear Vibrations in Structures With Contact Interfaces. fournal of Engineering for Gas Turbines and Power, 133(10):102503, 2011.

[32] F. Pourahmadian, H. Ahmadian, and H. Jalali. Modeling and identification of frictional forces at a contact interface experiencing micro-vibro-impacts. Fournal of Sound and Vibration, 331(12):2874 - 2886, 2012.

[33] C. W. Schwingshackl, E. P. Petrov, and D. J. Ewins. Measured and estimated friction interface parameters in a nonlinear dynamic analysis. Mechanical Systems and Signal Processing, 28:574-584, April 2012.

[34] J. Armand, L. Pesaresi, L. Salles, and C.W. Schwingshackl. A Multiscale Approach for Nonlinear Dynamic Response Predictions With Fretting Wear. Journal of Engineering for Gas Turbines and Power, 139(2), 2016.

[35] I. A. Polonsky and L. M. Keer. Fast methods for solving rough contact problems: A comparative study. f. Tribol., 122(1):36, 2000.

[36] K. L. Johnson. Contact Mechanics. Cambridge University Press, 1985. Cambridge Books Online.

[37] A. E. H. Love. A Treatise on the Mathematical Theory of Elasticity. A Treatise on the Mathematical Theory of Elasticity. at the University Press, 1906.

[38] C.W. Schwingshackl. Measurement of Friction Contact Parameters for Nonlinear Dynamic Analysis. In Proceedings of the Society for Experimental Mechanics IMAC Conference, 2012.
[39] R. H. Byrd, J. C. Gilbert, and J. Nocedal. A trust region method based on interior point techniques for nonlinear programming. Mathematical Programming, 89(1):149185, Nov 2000. 